\title{
La exclusión social bajo el enfoque de género en las comunidades campesinas de Copca y Santa Rosa de Roncha
}

\section{Social exclusion under the gender perspective in the rural communities of Copca and Santa Rosa de Roncha}

Cárdenas Espejo, Luis Gonzaga / Puchoc Cuestas, Esteban

Facultad de Economía de la Universidad Nacional del Centro del Perú

\section{RESUMEN}

En estas últimas décadas, muchos países del Tercer Mundo han alcanzado importantes tasas de crecimiento económico. Sin embargo, en muchos de ellos la tasa de pobreza se ha mantenido o ha disminuido de manera poco significativa. En este contexto, las sociedades sienten la necesidad de desarrollar determinadas estrategias de inclusión social que permitan alcanzar una mayor igualdad de oportunidades y la eliminación de las distancias en la participación social, económica y política entre hombres y mujeres.

En tal sentido, en la presente investigación sobre "La Exclusión Social Bajo un Enfoque de Género en las Comunidades Campesinas de Copca y Santa Rosa de Roncha", se buscó describir y comparar el comportamiento de las características de la exclusión social para determinar cómo es que se originan las diferencias de oportunidades entre hombres y mujeres, las interrelaciones existentes entre ellos y los distintos roles que socialmente se les asigna.

El método científico fue asumido como método general para el desarrollo de la investigación en referencia. Así mismo, se aplicaron como métodos particulares el deductivo e inductivo.

\section{ABSTRACT}

In recent decades, many Third World countries have achieved significant economic growth rates. However, in many of them the poverty rate has remained low or decreased significantly. In this context, companies feel the need to develop specific strategies for social inclusion that achieve greater equality of opportunity and the elimination of the distances in the social, economic and political participation between men and women.

In this sense, this research on "Social Exclusion Under a Gender Approach in Rural Communities in Copca and Santa Rosa de Roncha" sought to describe and compare the behavior of the characteristics of social exclusion to determine how it is to be opportunities arise differences between men and women, the interrelationships between them and the different roles that they are socially assigned.

The scientific method was assumed as a general method for the development of the research in question. Also, were applied as particular deductive and inductive methods.

Parametric Mann Whitney U test, chi square test and allowed to set as exclusion characteristics in the study area income and precarious employment. 
La prueba paramétrica U de Mann Whitney, y la prueba chi cuadrado permitieron establecer como características de exclusión en el área de estudio el nivel de ingreso y el empleo precario.

Como principal conclusión de la investigación desarrollada se tiene que en la comunidad de Copca el nivel de ingreso y el empleo precario constituyen características de exclusión social. Mientras que en la comunidad de Roncha, sólo el nivel de ingreso constituye una característica de exclusión social.

Palabras clave: Enfoque de género, exclusión, empleo precario, concentración de ingreso, servicios básicos, roles de la mujer y el hombre, grupos vulnerables.
The main conclusion of the research is to be developed in the community of Copca income level and precarious employment are characteristics of social exclusion. While in the community Roncha, only income is a characteristic of social exclusion.

Keywords: Gender approach, exclusion, precarious employment, income concentration, basic services, roles of women and men, vulnerable groups.

\section{INTRODUCCIÓN}

En estos últimos años el problema de la pobreza ha venido siendo abordado desde la perspectiva de la inclusión social. Un buen número de investigaciones en el Perú han tocado el tema de la exclusión social en el área urbana vinculándola mayormente al tema económico o al crecimiento económico del país.

Con la investigación sobre "La Exclusión Social Bajo el Enfoque de Género en las Comunidades Campesinas de Copca y Santa Rosa de Roncha", se busca contribuir a la discusión sobre la exclusión social en el área rural.

Uno de los aspectos centrales en el tratamiento sobre la exclusión social es precisamente partir entendiendo que el desarrollo humano constituye un proceso de ampliación de las opciones que tienen las personas de vivir una vida prolongada y saludable, adquirir valores y conocimientos, desarrollar destrezas y tener acceso a los recursos necesarios para lograr una alta calidad de vida.

En este trabajo de investigación se busca describir comparativamente el problema de la exclusión social a partir de aspectos vinculados con el comportamiento económico, institucional y social basado en las diferencias de género. En tal sentido, se especificó como características de exclusión social, el nivel de ingreso, el empleo precario, los servicios básicos, los roles del hombre y de la mujer, y los grupos vulnerables.

A partir de la descripción comparativa de las características de la exclusión social, se buscó su particularización en el área rural de estudio en cuanto a su comportamiento como un fenómeno multidisciplinario y transversal.

Los resultados del presente trabajo de investigación, consideramos que podrían ser tomados en cuenta por los gobiernos locales y gobierno regional como insumos para el diseño de políticas de inclusión social y lucha contra la pobreza.

\section{MATERIAL Y MÉTODOS}

\section{Metodología}

Tipo y nivel de investigación: Aplicada y descriptiva.

\section{Métodos de Investigación}

El método científico fue asumido como método general para el desarrollo del presente trabajo de investigación. Asimismo, se emplearon los 
siguientes métodos particulares: el inductivo, porque permitió analizar el comportamiento de cada una de las variables propuestas como descriptivas del problema de investigación; $y$, el inductivo, por que partiendo de determinados principios y propuestas teóricas consintió confirmar las conclusiones alcanzadas al término de la investigación mediante el procedimiento de la deducción.

\section{Diseño metodológico}

a) Población y Muestra: La población del área de estudio estuvo constituida por los comuneros de Copca y Santa Rosa de Roncha inscritos en el padrón comunal.

En cuanto al tamaño de la muestra poblacional, fue determinada del siguiente modo:
Comunidad Campesina de Copca

Población: 34 comuneros activos

Tamaño de la muestra poblacional:

$\mathrm{n}=\mathrm{Z}^{2}(\mathrm{p})(\mathrm{q}) \mathrm{N} / \mathrm{e}^{2}(\mathrm{~N}-1)+\mathrm{Z}^{2}(\mathrm{p})(\mathrm{q})$

Donde:

N: Población bajo estudio

$\mathrm{n}$ : Tamaño de muestra

Z: Nivel de confianza (95\%)

p: Probabilidad que el evento ocurra

q: Probabilidad que el evento no ocurra

e: Error de estimación (5\%)

$\mathrm{n}=33$

\section{Comunidad Campesina de Santa Rosa de Ron-} cha

Población: 40 comuneros activos

$\mathrm{n}=36$

\section{RESULTADOS}

\section{Nivel de Ingreso}

Tabla 1. Jornal diario del encuestado como peón agrícola, según comunidad campesina y género

\begin{tabular}{cccccc}
\hline \multirow{2}{*}{ Género } & \multirow{2}{*}{ Jornal diario } & \multicolumn{2}{c}{ SR de Roncha } & \multicolumn{2}{c}{ Copca } \\
\cline { 2 - 6 } & No trabaja & Encuestados & $\%$ & Encuestados & $\%$ \\
\hline \multirow{4}{*}{ Varón } & 40 & 3 & 44.4 & 11 & 47.8 \\
& 45 & 7 & 16.7 & 6 & 26.1 \\
& 50 & & 38.9 & 5 & 21.7 \\
& Total & 18 & 100.0 & 1 & 4.3 \\
\multirow{4}{*}{ Mujer } & No trabaja & 5 & 27.8 & 23 & 100.0 \\
& 25 & 5 & 27.8 & 5 & 41.7 \\
& 30 & 4 & 22.2 & 2 & 16.7 \\
& 35 & 4 & 22.2 & 3 & 25.0 \\
& 40 & & & 1 & 8.3 \\
& 45 & & & 1 & 8.3 \\
& Total & 18 & 100,0 & 12 & 100.0 \\
\hline
\end{tabular}

Jornal promedio: Roncha: Varones $=43.50 ;$ Mujeres $=29.62 ;$ Copca: Varones $=42.92 ;$ Mujeres $=35.17$

Roncha: Valor $\mathrm{p}$ (Shapiro Wilk): Varones $=0$; Mujeres $=0.007$; Valor $\mathrm{p}(\mathrm{U}$ de Mann Whitney) $=0$

Copca: Valor $\mathrm{p}$ (Shapiro Wilk): Varones $=0.004$; Mujeres $=0.294 ;$ Valor $p(U$ de Mann Whitney) $=0.007$

En la tabla 1 y figura 1 , se observa que en la comunidad de Santa Rosa de Roncha, el jornal diario como peón agrícola de los varones encuestados oscila entre 40 y 45 nuevos soles y de las mujeres entre 25 y 35 nuevos soles, donde el $38.9 \%$ de varones ganan 45 nuevos soles y el $27.8 \%$ de mujeres 25 nuevos soles. El jornal promedio de los varones encuestados es de $\mathrm{S} /$. 43.50 y de las mujeres S/. 29.62. 
En la comunidad de Copca, el jornal diario como peón agrícola de los varones encuestados oscila entre 40 y 50 nuevos soles y de las mujeres entre 30 y 45 nuevos soles, donde el
$26.1 \%$ de los varones ganan 40 nuevos soles y el $25 \%$ de las mujeres 35 nuevos soles. El jornal promedio de los varones encuestados es de S/. 42.92 y de las mujeres S/. 35.17

Figura 1.

\section{Jornal diario del encuestado como peón agrícola, según comunidad campesina y género}

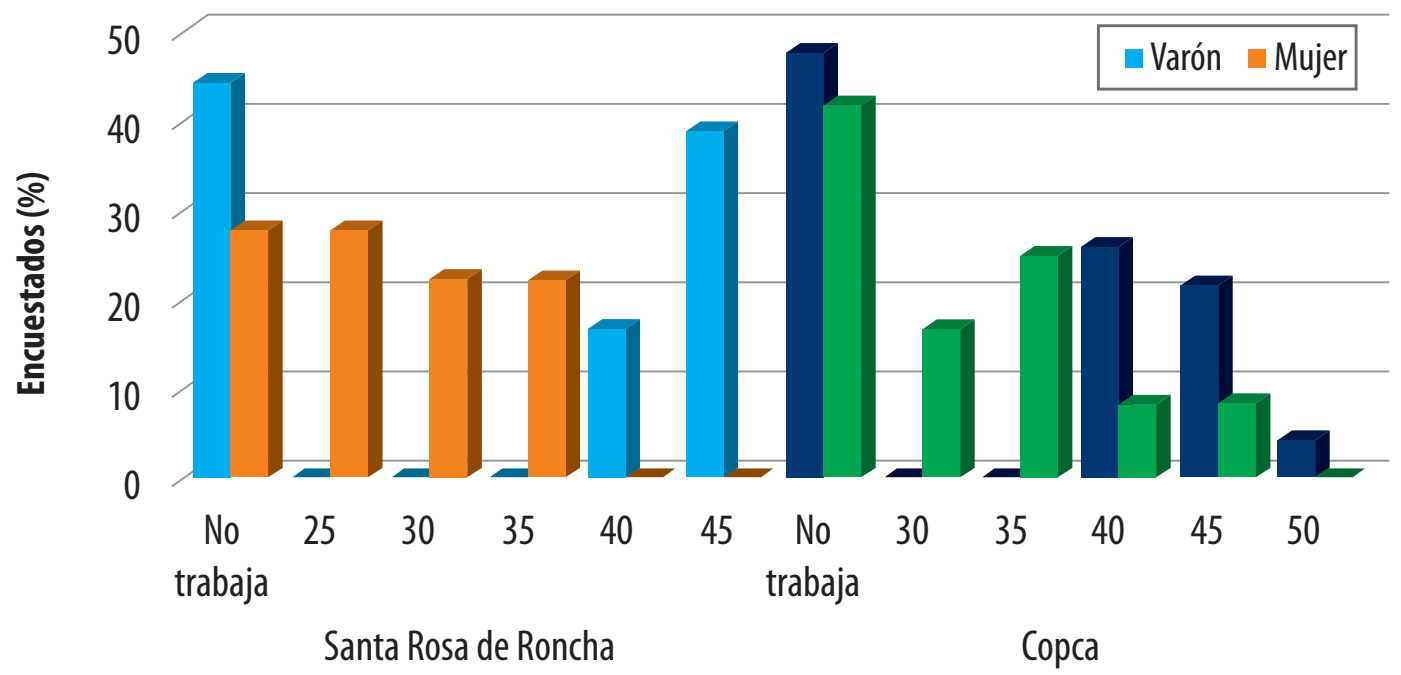

La prueba de normalidad de Shapiro Wilk revela que las distribuciones del jornal diario como peón agrícola de los varones y mujeres encuestadas de la comunidad de Santa Rosa de Roncha son no normales $(p<0.05)$. La distribución del jornal diario como peón agrícola de los varones encuestados de la comunidad de Copca es no normal ( $p<0.05)$, mientras que la distribución del jornal de las mujeres si lo es $(p>0.05)$.
La prueba no paramétrica $U$ de Mann Whitney para la homogeneidad de muestras revela que en ambas comunidades, existen diferencias significativas en las medias o medianas del jornal diario de los varones y mujeres encuestadas como peón agrícola, favorables a los varones de acuerdo con la información de los varones y mujeres $(p<0.05)$. 


\section{Empleo Precario}

Tabla 2. Ocupación del encuestado, según comunidad campesina y género

\begin{tabular}{|c|c|c|c|c|c|}
\hline \multirow{2}{*}{ Género } & \multirow{2}{*}{ Ocupación } & \multicolumn{2}{|c|}{ SR de Roncha } & \multicolumn{2}{|c|}{ Copca } \\
\hline & & Encuestados & $\%$ & Encuestados & $\%$ \\
\hline \multirow{5}{*}{ Varón } & Agricultor & 13 & 72.2 & 14 & 60.9 \\
\hline & Ganadero & & & 9 & 39.1 \\
\hline & Peón agrícola & 3 & 16.7 & & \\
\hline & Comerciante & 2 & 11.1 & & \\
\hline & Total & 18 & 100.0 & 23 & 100.0 \\
\hline \multirow{5}{*}{ Mujer } & Agricultor & 14 & 77.8 & 3 & 25.0 \\
\hline & Ganadero & & & 9 & 75.0 \\
\hline & Peón agrícola & 3 & 16.7 & & \\
\hline & Comerciante & 1 & 5.6 & & \\
\hline & Total & 18 & 100.0 & 12 & 100.0 \\
\hline
\end{tabular}

Roncha: Estadístico chi cuadrado $=0 ;$ Valor $p=1$

Copca: Estadístico chi cuadrado $=37,259 ;$ Valor $p=0$

En la tabla 2 y figura 2, se tiene que en la comunidad de Santa Rosa de Roncha, la mayoría de varones $(72.2 \%)$ y mujeres $(77.8 \%)$ encuestados son agricultores.
En la comunidad de Copca, la mayoría de varones $(60.9 \%)$ encuestados son agricultores y la mayoría de mujeres (75\%) entrevistadas son ganaderas.

Figura 2.

Ocupación del encuestado, según comunidad campesina y género

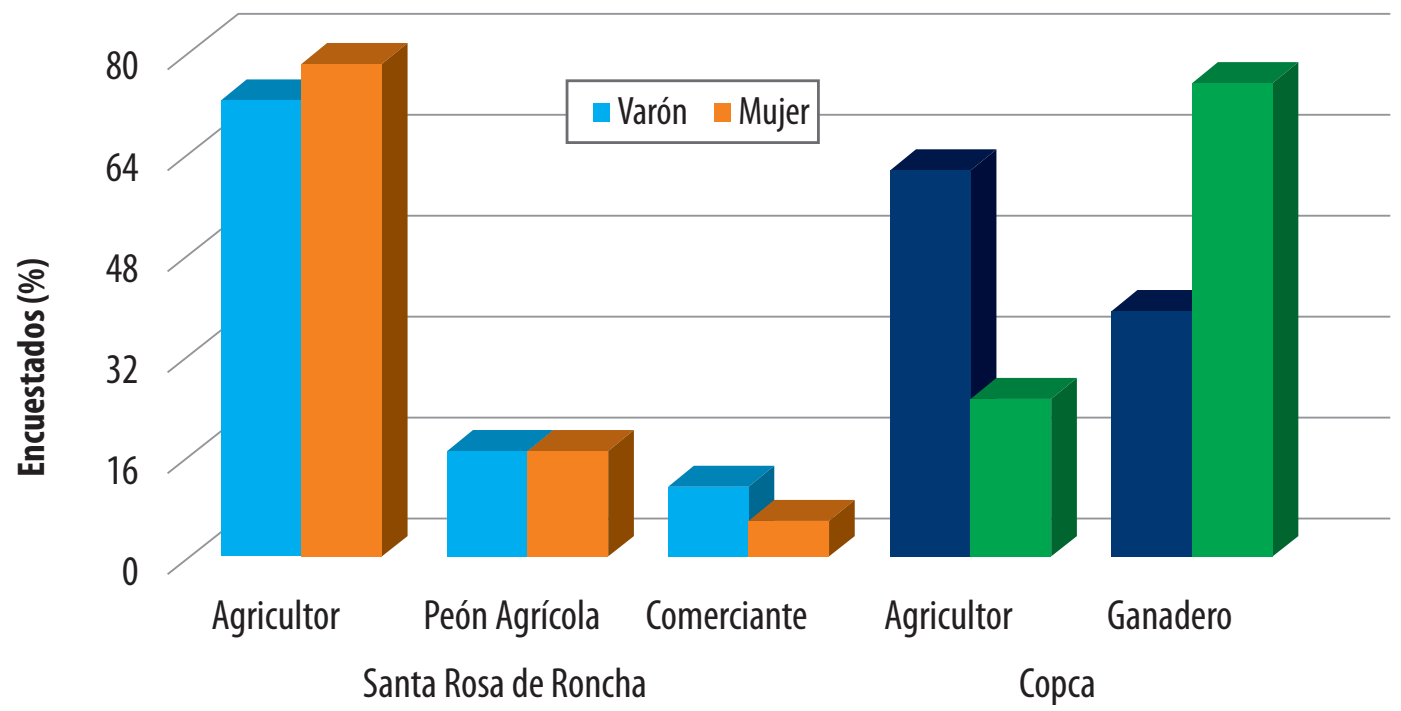

De otro lado, se aprecia que el empleo precario (agricultor y peón agrícola) de los varones de la comunidad de Santa Rosa de Roncha es del orden del $88.9 \%$ frente al $94.4 \%$ de las mujeres de la comunidad en referencia.

También, que el empleo precario de los varones de la comunidad de Copca alcanza el $60.9 \%$ 
frente al $25 \%$ de las mujeres de dicha comunidad.

La prueba chi cuadrado para la homogeneidad de muestras revela que en la comunidad de Santa Rosa de Roncha, no existen diferencias significativas en las proporciones del empleo precario de los encuestados de acuerdo con la información de los varones y mujeres $(p>$ 0.05).

No obstante, esta prueba indica que en la comunidad de Copca, si existen diferencias significativas en las proporciones del empleo precario de los encuestados de acuerdo con la información de los varones y mujeres $(p<0.05)$.

\section{Servicios Básicos}

Tabla 3. Material predominante en la vivienda de los encuestados, según comunidad campesina y género

\begin{tabular}{clcccr}
\hline \multirow{2}{*}{ Género } & \multirow{2}{*}{ Material } & \multicolumn{2}{c}{ SR de Roncha } & \multicolumn{2}{c}{ Copca } \\
\cline { 2 - 6 } & Adobe & Encuestados & $\%$ & Encuestados & $\%$ \\
\hline \multirow{4}{*}{ Varón } & 17 & 94.4 & 19 & 82.6 \\
& Noble & 1 & 5.6 & 3 & 13.0 \\
& Quincha & & & 1 & 4.3 \\
& Total & 18 & 100.0 & 23 & 100.0 \\
\hline \multirow{6}{*}{ Mujer } & Adobe & 15 & 83.3 & 8 & 66.7 \\
& Noble & 2 & 11.1 & 1 & 8.3 \\
& Quincha & 1 & 5.6 & 3 & 25.0 \\
& Total & 18 & 100.0 & 12 & 100.0 \\
\hline
\end{tabular}

Roncha: Estadístico chi cuadrado $=0 ;$ Valor $\mathrm{p}=1$

Copca: Estadístico chi cuadrado $=0 ;$ Valor $p=1$

Figura 3.

\section{Material predominante en la vivienda de los encuestados, según comunidad campesina y género}

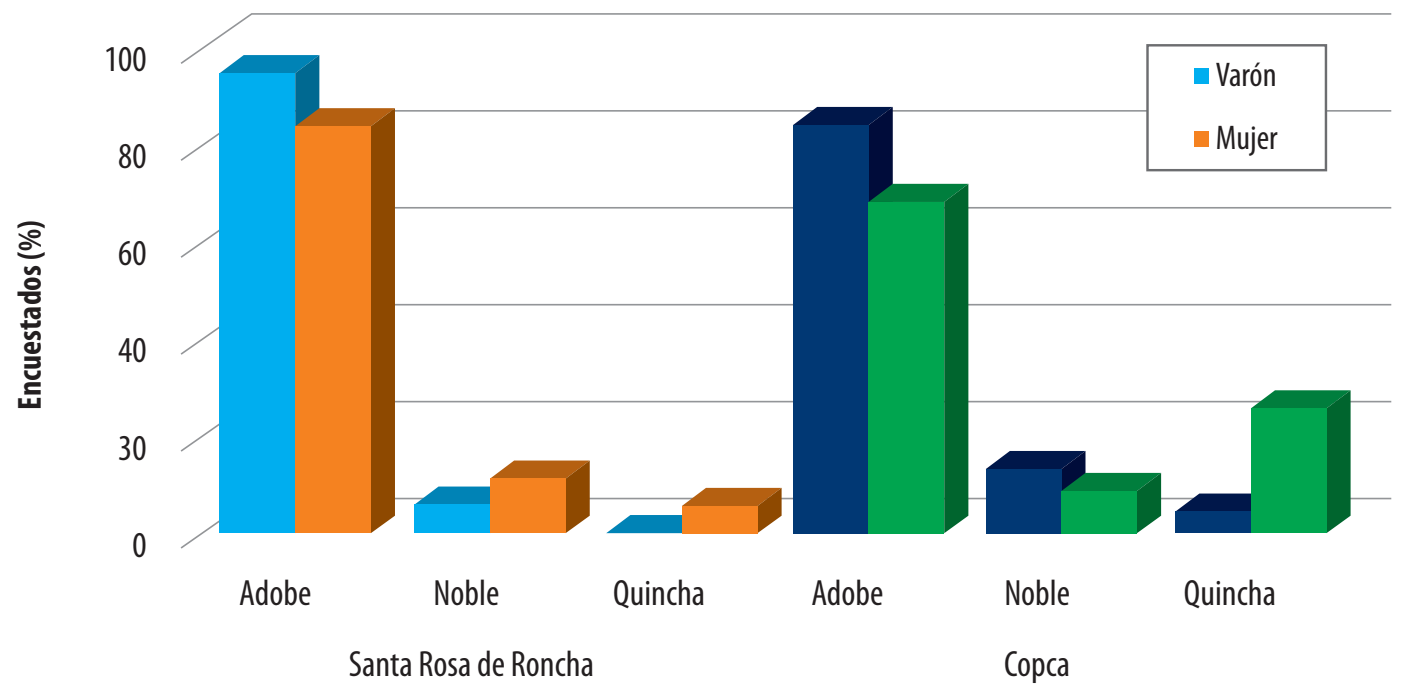


En la tabla 3 y figura 3 , se valora que la calidad baja (adobe, quincha) de la vivienda de los varones de la comunidad de Santa Rosa de Roncha es del orden del $94.4 \%$ frente al $88.9 \%$ de las mujeres de esta comunidad. Igualmente, que la calidad baja de la vivienda de los varones de la comunidad de Copca alcanza el $87 \%$ frente al $91.7 \%$ de las mujeres de la comunidad en referencia.

La prueba chi cuadrado para la homogeneidad de muestras revela que en ambas comunida- des, no existen diferencias significativas en las proporciones de viviendas de calidad baja de los encuestados, de acuerdo con la información de los varones y mujeres ( $p>0.05$ ).

Estos resultados indican que la calidad baja de las viviendas no es una característica de exclusión social bajo un enfoque de género en las comunidades de Santa Rosa de Roncha y Copca, con lo cual se rechaza la hipótesis de investigación en lo que se refiere a la calidad baja de las viviendas.

Tabla 4. Servicios de la vivienda de los encuestados, según comunidad campesina y género

\begin{tabular}{llcrcr}
\hline \multirow{2}{*}{ Género } & \multirow{2}{*}{ Servicio } & \multicolumn{2}{c}{ SR de Roncha } & \multicolumn{2}{c}{ Copca } \\
\cline { 2 - 6 } Varón & Encuestados & \multicolumn{1}{c}{$\%$} & Encuestados & $\%$ \\
\cline { 2 - 6 } & Luz & 5 & 27.8 & 3 & 13.0 \\
& Agua & 7 & 38.9 & & \\
& Luz y agua & 5 & 27.8 & 20 & 87.0 \\
& Agua y pozo & 1 & 5.6 & & \\
& Total & 18 & 100.0 & 23 & 100.0 \\
\hline \multirow{6}{*}{ Mujer } & Luz & 7 & 38.9 & 1 & 8.3 \\
& Agua & 5 & 27.8 & 1 & 8.3 \\
& Luz y agua & 5 & 27.8 & 10 & 83.3 \\
& Agua y desagüe & 1 & 5.6 & & \\
& Total & 18 & 100.0 & 12 & 100.0 \\
\hline
\end{tabular}

Roncha: Estadístico chi cuadrado $=0 ;$ Valor $p=1$

Copca: Estadístico chi cuadrado $=0 ;$ Valor $p=1$

Figura 4.

\section{Servicio de la vivienda de los encuestados, según comunidad campesina y género}

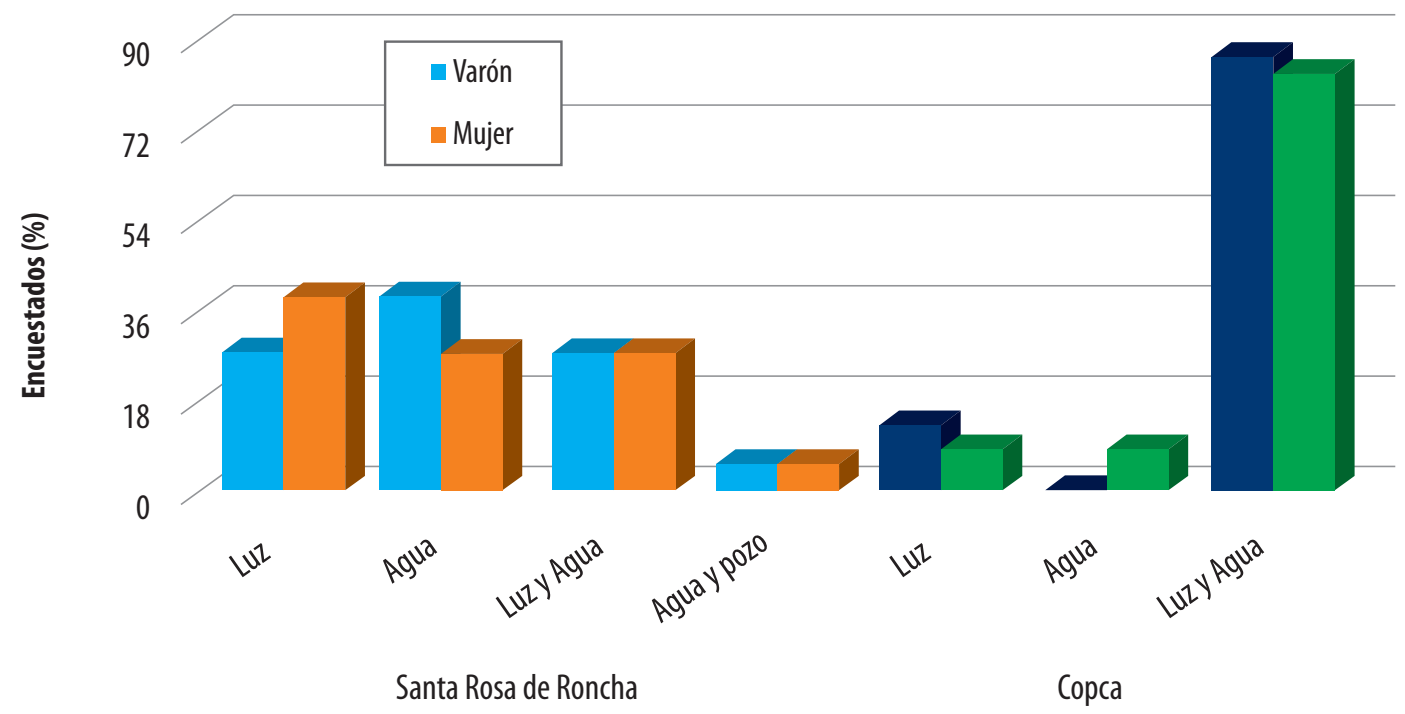


En la tabla 4 y figura 4, se establece que en la comunidad de Santa Rosa de Roncha, el 38.9\% de varones y el $27.8 \%$ de mujeres entrevistadas tienen vivienda con servicio de agua, el $27.8 \%$ de varones y el $38.9 \%$ de mujeres poseen vivienda con servicio de luz, el $27.8 \%$ de los primeros y de las segundas tienen vivienda con luz y agua.

En la comunidad de Copca, la mayoría de varones (87\%) y mujeres (83.3\%) encuestadas tienen vivienda con servicio de luz y agua.

Por otra parte, se aprecia que la carencia o falta de servicios básicos (agua y luz, en forma conjunta, por ser una zona rural) en la vivienda de los varones de la comunidad de Santa Rosa de Roncha es del orden del $72.2 \%$ frente al $72.2 \%$ de las mujeres de esta comunidad. Por su lado, la falta de servicios básicos en las viviendas de los varones de la comunidad de Copca alcanza el $13 \%$ frente al $16.7 \%$ de las mujeres de esta comunidad.

La prueba chi cuadrado para la homogeneidad de muestras revela que en ambas comunidades, no existen diferencias significativas en las proporciones de la carencia de servicios básicos en la vivienda de los encuestados de acuerdo con la información de los varones y mujeres ( $p$ $>0.05)$.

Estos resultados indican que la falta de servicios básicos en la vivienda no es una característica de exclusión social bajo un enfoque de género en las comunidades de Santa Rosa de Rocha y Copca, con lo cual se rechaza la hipótesis de investigación, en lo que se refiere a la falta de servicios básicos en la vivienda.

Tabla 5. Índice de hacinamiento en la vivienda de los encuestados, según comunidad campesina y género

\begin{tabular}{clcccc}
\hline \multirow{2}{*}{ Género } & Indice de & \multicolumn{2}{c}{ SR de Roncha } & \multicolumn{2}{c}{ Copca } \\
\cline { 2 - 6 } & hacinamiento & Encuestados & $\%$ & Encuestados & $\%$ \\
\hline \multirow{6}{*}{ Varón } & $<1$ & 1 & 5.6 & 1 & 4.3 \\
& 1 a 1.99 & 7 & 38.9 & 8 & 34.8 \\
& 2 a + & & & 10 & 43.5 \\
& No informa & 10 & 55.6 & 4 & 17.4 \\
& Total & 18 & 100.0 & 23 & 100.0 \\
\hline \multirow{6}{*}{ Mujer } & $<1$ & 1 & 5.6 & 2 & 16.7 \\
& 1 a 1.99 & 8 & 44.4 & 4 & 33.3 \\
& 2 a + & 1 & 5.6 & 2 & 16.7 \\
& No informa & 8 & 44.4 & 4 & 33.3 \\
& Total & 18 & 100.0 & 12 & 100.0 \\
\hline
\end{tabular}

Indice de hacinamiento promedio:

Roncha: Varones = 1.17; Mujeres = 1.29; Copca: Varones = 1.82; Mujeres = 1,54

Roncha: Valor $\mathrm{p}$ (Shapiro Wilk): Varones $=0.452$; Mujeres $=0.344$; Valor $\mathrm{p}(\mathrm{F}$ de Fisher) $=0.885 ;$ Valor $\mathrm{p}(\mathrm{t}$ de Student) $=0.304$

Copca: Valor $\mathrm{p}$ (Shapiro Wilk): Varones $=0.431$; Mujeres $=0.534 ;$ Valor $\mathrm{p}(\mathrm{F}$ de Fisher $)=0.613 ;$ Valor $\mathrm{p}(\mathrm{t}$ de Student) $=0.50$

En la tabla 5 se aprecia que el $38.9 \%$ de las viviendas de los varones de la comunidad de Santa Rosa de Roncha alcanza un índice de hacinamiento de 1 a 1.99 personas por habitación, frente al $44.4 \%$ de las viviendas de las mujeres de esta comunidad. Por su lado, el $43.5 \%$ de las viviendas de los varones de la comunidad de Copca alcanza un índice de hacinamiento de 2 a más personas por habitación, frente al
$16.7 \%$ de las viviendas de las mujeres de esta comunidad.

En la comunidad de Santa Rosa de Roncha, el índice promedio de hacinamiento en las viviendas de los varones y mujeres son de 1.17 y 1.29 personas por habitación. Por su parte, en la comunidad de Copca, este índice es de 1.82 y 1.54 personas por habitación en las viviendas 
de los varones y mujeres, respectivamente.

La prueba de normalidad de Shapiro Wilk revela que las distribuciones del índice de hacinamiento en las viviendas de los varones y mujeres encuestadas de ambas comunidades son normales ( $p>0.05)$.

La prueba paramétrica de la $\mathrm{F}$ de Fisher para la homogeneidad de varianzas indica que en ambas comunidades no hay diferencias significativas entre las varianzas del índice de hacinamiento en las viviendas de los varones y mujeres encuestadas ( $p>0.05$ ). Por su parte, la prueba t de Student para la diferencia de medias de dos muestras independientes pequeñas revela que no existen diferencias significativas entre las medias del índice de hacinamiento en las viviendas de los varones y mujeres entrevistadas $(p>0.05)$.

Estos resultados indican que el índice de hacinamiento en la vivienda no es una característica de exclusión social bajo un enfoque de género en las comunidades de Santa Rosa de Roncha y Copca, con lo cual se rechaza la hipótesis de investigación en lo que se refiere al índice de hacinamiento en la vivienda.

\section{Roles de la Mujer y del Varón}

Tabla 6. Persona que cuida el hogar, según comunidad y campesina y género

\begin{tabular}{llcrcr}
\hline \multirow{2}{*}{ Género } & \multirow{2}{*}{ Persona } & \multicolumn{2}{c}{ SR de Roncha } & \multicolumn{2}{c}{ Copca } \\
\cline { 2 - 6 } & Encuestados & $\%$ & Encuestados & $\%$ \\
\hline \multirow{4}{*}{ Varón } & 5 & 27.8 & 3 & 13.0 \\
& La mujer & 11 & 61.1 & 19 & 82.6 \\
& Otros familiares & 2 & 11.1 & 1 & 4.3 \\
& Total & 18 & 100.0 & 23 & 100.0 \\
\hline \multirow{4}{*}{ Mujer } & El varón & 2 & 11.1 & 3 & 25.0 \\
& La mujer & 15 & 83.3 & 9 & 75.0 \\
& Otros familiares & 1 & 5.6 & & 100.0 \\
& Total & 18 & 100.0 & 12 &
\end{tabular}

Roncha: Estadístico chi cuadrado $=0.888$; Valor $p=0.346$

Copca: Estadístico chi cuadrado $=0.130 ;$ Valor $p=0.719$

En la tabla 6 y figura 6, se aprecia que en la comunidad de Santa Rosa de Roncha, la mayoría de varones $(61.1 \%)$ y mujeres $(83.3 \%)$ encuestadas informan que la mujer cuida el hogar, el $27.8 \%$ de varones y el $11.1 \%$ de mujeres manifiestan que lo hace el varón.
De igual manera, en la comunidad de Copca, la mayoría de varones $(82.6 \%)$ y mujeres $(75 \%)$ entrevistadas, afirman que la mujer cuida el hogar, el $13 \%$ de varones y el $25 \%$ de mujeres sostienen que lo hace el varón. 
Figura 6.

\section{Persona que cuida el hogar, según comunidad campesina y género}

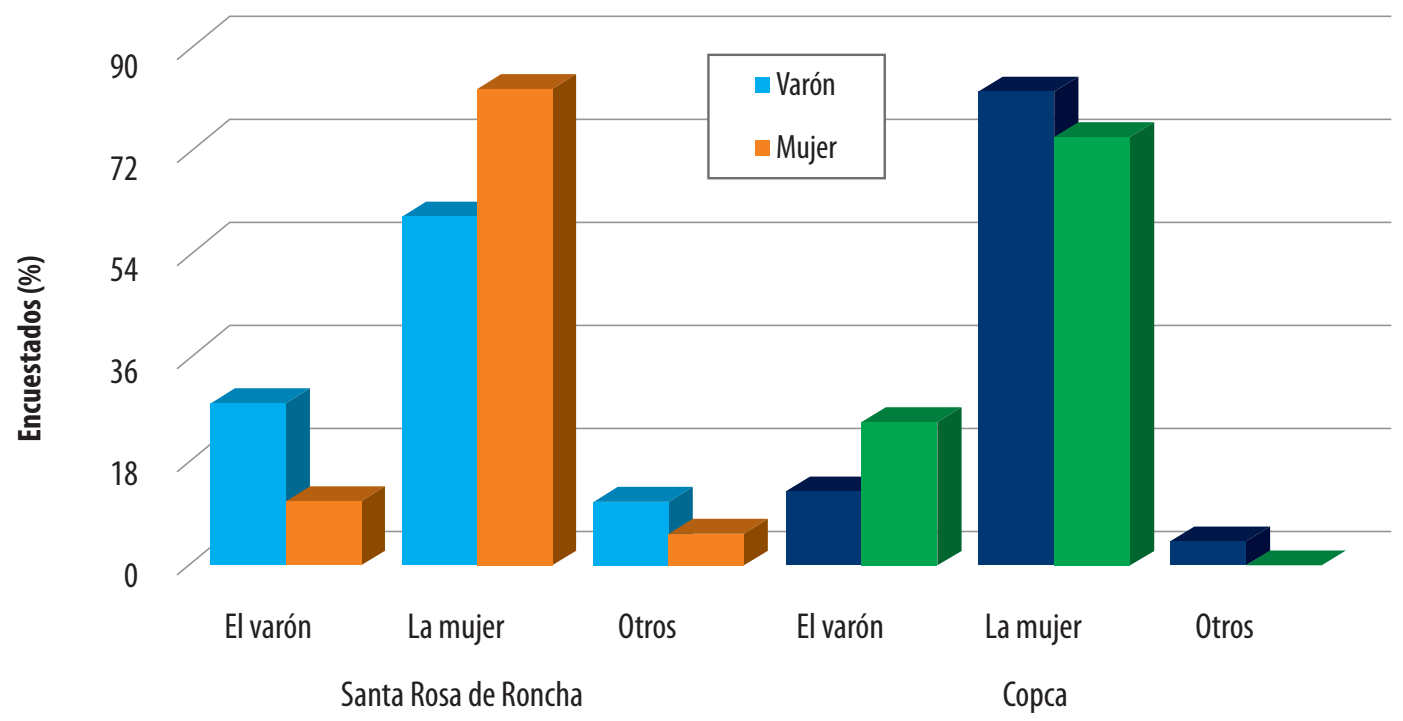

Así mismo, en la comunidad de Santa Rosa de Roncha, el cuidado del hogar está a cargo de la mujer para el $61.1 \%$ de los varones y el $83.3 \%$ de las mujeres encuestadas. En la comunidad de Copca, el cuidado del hogar también está a cargo de la mujer para el $82.6 \%$ de los varones y el $75 \%$ de las mujeres entrevistadas.

La prueba chi cuadrado para la homogeneidad de muestras revela que en ambas comunidades no existen diferencias significativas en las pro- porciones de las personas que cuidan el hogar, de acuerdo con la información de los varones y mujeres $(p>0.05)$.

Estos resultados indican que el rol de la mujer y el varón en el cuidado del hogar no es una característica de exclusión social bajo un enfoque de género en las comunidades de Santa Rosa de Roncha y Copca, con lo cual se rechaza la hipótesis de investigación en lo que se refiere a los roles de la mujer y el varón en el hogar.

Tabla 7. Persona que cuida los hijos, según comunidad y campesina y género

\begin{tabular}{llcrrr}
\hline \multirow{2}{*}{ Género } & \multirow{2}{*}{ Persona } & \multicolumn{2}{c}{ SR de Roncha } & \multicolumn{2}{c}{ Copca } \\
\cline { 2 - 6 } & El varón & 1 & $\%$ & Encuestados & $\%$ \\
\hline \multirow{4}{*}{ Varón } & La mujer & 16 & 88.9 & 1 & 4.3 \\
& Otros familiares & 1 & 5.6 & 18 & 78.3 \\
& Total & 18 & 100.0 & 23 & 17.4 \\
& La mujer & 17 & 94.4 & 11 & 91.7 \\
\multirow{3}{*}{ Mujer } & Otros familiares & 1 & 5.6 & 1 & 8.3 \\
& Total & 18 & 100.0 & 12 & 100.0 \\
\hline
\end{tabular}

Roncha: Estadístico chi cuadrado $=0 ;$ Valor $p=1$

Copca: Estadístico chi cuadrado $=0 ;$ Valor $p=1$ 
De igual manera, en la comunidad de Copca la mayoría de varones $(78.3 \%)$ y mujeres $(91.7 \%)$ entrevistadas afirman que la mujer cuida los hi- jos, el $17.4 \%$ de varones y el $8.3 \%$ de mujeres sostienen que lo hacen otros familiares.

Figura 7.

\section{Persona que cuida los hijos, según comunidad campesina y género}

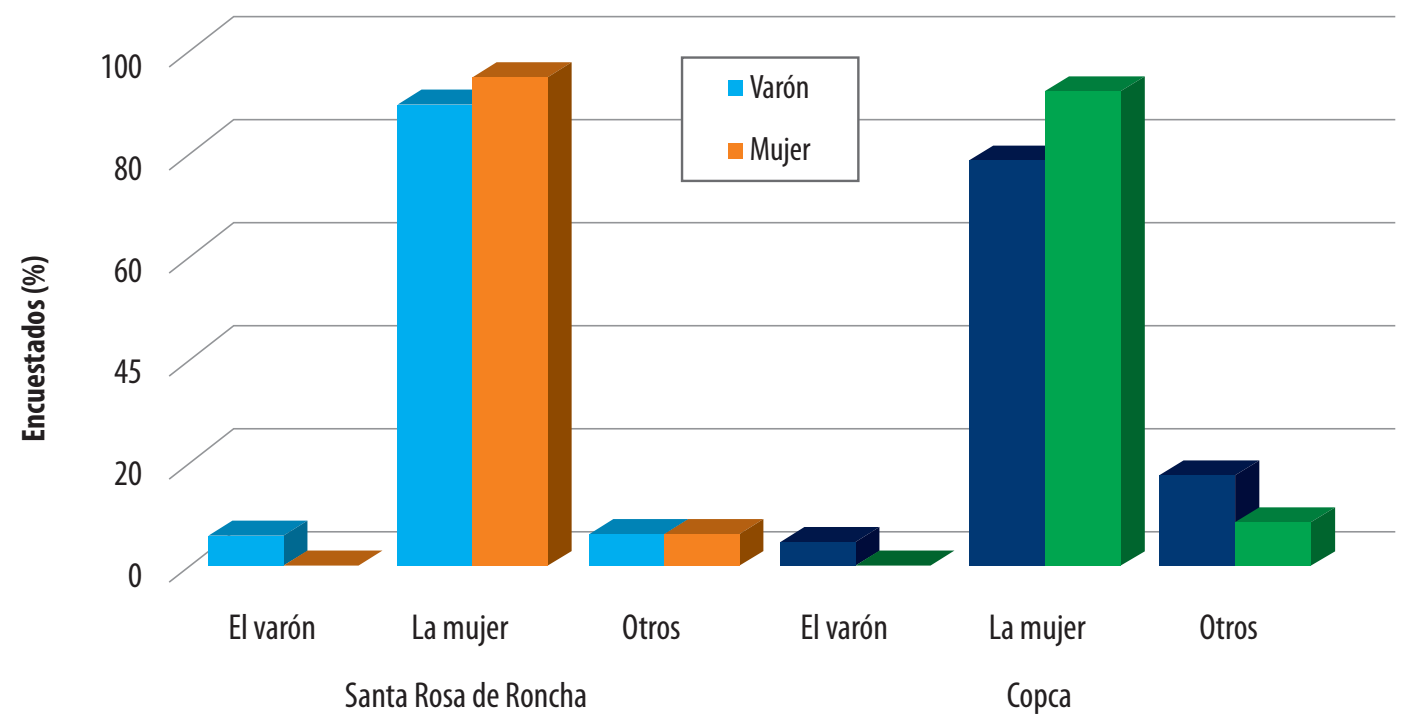

De otro lado, en la comunidad de Santa Rosa de Roncha, el cuidado de los hijos está a cargo de la mujer para el $88.9 \%$ de los varones y el $94.4 \%$ de las mujeres encuestadas. En la comunidad de Copca, el cuidado de los hijos también está a cargo de la mujer para el 78.3\% de los varones y el $\mathbf{9 1 . 7 \%}$ de las mujeres entrevistadas.

La prueba chi cuadrado para la homogeneidad de muestras revela que en ambas comunidades no existen diferencias significativas en las pro- porciones de las personas que cuidan los hijos, de acuerdo con la información de los varones y mujeres $(p>0.05)$.

Estos resultados indican que el rol de la mujer y el varón en el cuidado de los hijos no es una característica de exclusión social bajo un enfoque de género en las comunidades de Santa Rosa de Roncha y Copca, con lo cual se rechaza la hipótesis de investigación en lo que se refiere a los roles de la mujer y el varón en el hogar. 
Tabla 8. Desempeño de algún cargo por el encuestado, según comunidad y campesina y género

\begin{tabular}{clcccr}
\hline \multirow{2}{*}{ Género } & \multirow{2}{*}{ Desempeño } & \multicolumn{2}{c}{ SR de Roncha } & \multicolumn{2}{c}{ Copca } \\
\cline { 3 - 6 } & & Encuestados & $\%$ & Encuestados & $\%$ \\
\hline \multirow{3}{*}{ Varón } & $\mathrm{Si}$ & 4 & 22.2 & 1 & 4.3 \\
& $\mathrm{No}$ & 14 & 77.8 & 22 & 95.7 \\
& Total & 18 & 100.0 & 23 & 100.0 \\
\hline \multirow{3}{*}{ Mujer } & $\mathrm{Si}$ & 2 & 11.1 & & \\
& No & 16 & 88.9 & 12 & 100.0 \\
& Total & 18 & 100.0 & 12 & 100.0 \\
\hline
\end{tabular}

Roncha: Estadístico chi cuadrado $=0,8 ;$ Valor $p=0,371$

Copca: Estadístico chi cuadrado $=0,537$; Valor $p=0,464$

En la tabla 8 y figura 8 , se observa que en la comunidad de Santa Rosa de Roncha, el 22.2\% de varones y el $11.1 \%$ de mujeres entrevistadas desempeñan algún cargo en su comunidad (pre- sidente, secretario o vocal).

En la comunidad de Copca, el $4.3 \%$ de varones y ninguna mujer encuestada desempeñan algún cargo en su comunidad.

Figura 8

\section{Desempeño de algún cargo por el encuestado, según comunidad campesina y género}

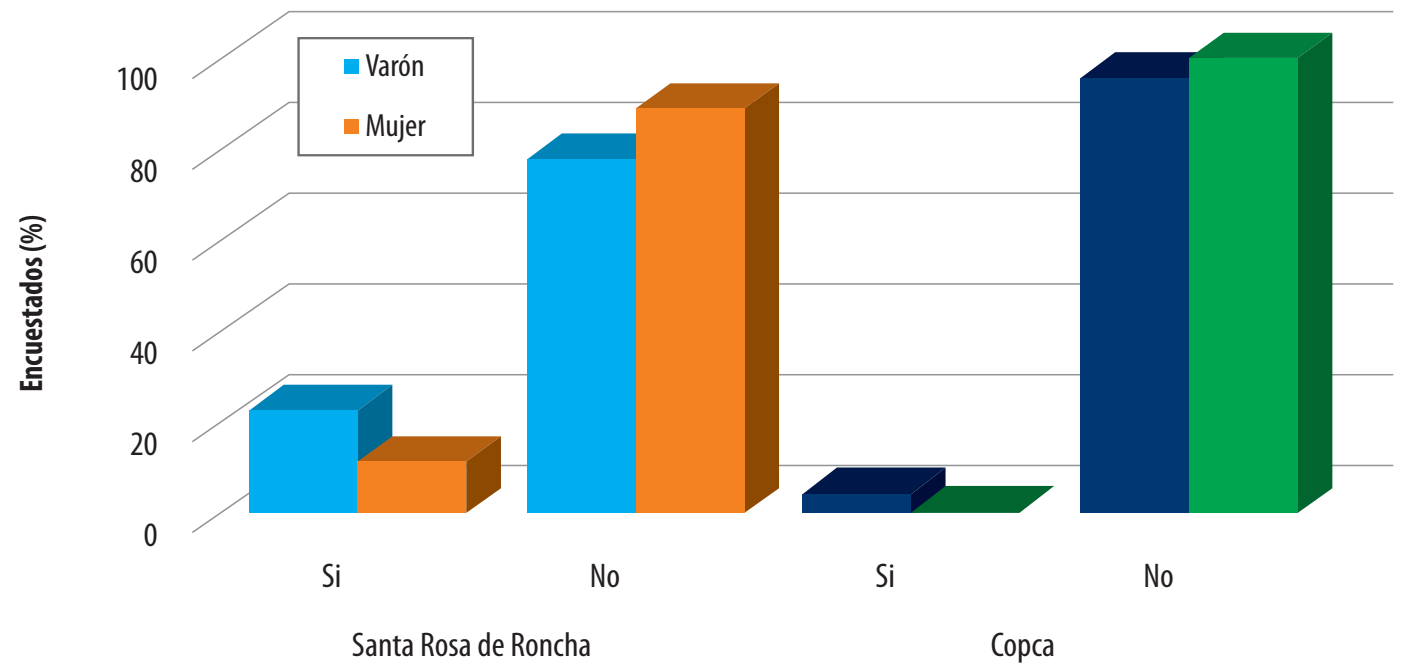

La prueba chi cuadrado para la homogeneidad de muestras revela que en ambas comunidades no existen diferencias significativas en las proporciones de desempeño de algún cargo en la comunidad de los encuestados, de acuerdo con la información de los varones y mujeres ( $p$ $>0.05)$.
Estos resultados indican que el rol de la mujer y el varón en la sociedad no es una característica de exclusión social bajo un enfoque de género en las comunidades de Santa Rosa de Roncha y Copca, con lo cual se rechaza la hipótesis de investigación en lo que se refiere a los roles de la mujer y el varón en la sociedad. 


\section{Grupos Vulnerables}

Tabla 9. Persona que cuida los niños, según comunidad y campesina y género

\begin{tabular}{clcccc}
\hline \multirow{2}{*}{ Género } & \multirow{2}{*}{ Persona } & \multicolumn{2}{c}{ SR de Roncha } & \multicolumn{2}{c}{ Copca } \\
\cline { 2 - 6 } & Sin niños & Encuestados & $\%$ & Encuestados & $\%$ \\
\hline \multirow{4}{*}{ Varón } & El varón & 4 & 22.2 & 4 & 17.4 \\
& La mujer & 13 & 72.2 & 10 & 43.5 \\
& Otros & & & 9 & 39.1 \\
& Total & 18 & 100.0 & 23 & 100.0 \\
\hline \multirow{4}{*}{ Mujer } & Sin niños & 1 & 5.6 & 2 & 16.7 \\
& La mujer & 17 & 94.4 & 6 & 50.0 \\
& Otros & & & 4 & 33.3 \\
& Total & 18 & 100.0 & 12 & 100.0 \\
\hline
\end{tabular}

Roncha: Estadístico chi cuadrado $=4.533 ;$ Valor $\mathrm{p}=0.104$

Copca: Estadístico chi cuadrado $=0.147 ;$ Valor $\mathrm{p}=0.929$

En la tabla 9 y figura 9, se tiene que en la comunidad de Santa Rosa de Roncha, la mayoría de varones $(72.2 \%)$ y mujeres encuestadas $(94.4 \%)$ informan que la mujer cuida a los niños.

En la comunidad de Copca, el $43.5 \%$ de varones y el $50 \%$ de mujeres entrevistadas manifiestan que la mujer cuida a los niños.

Figura 9.
La prueba chi cuadrado para la homogeneidad de muestras revela que en ambas comunidades no existen diferencias significativas en las proporciones de la persona que cuida a los niños en los hogares de los entrevistados, de acuerdo con la información de los varones y mujeres ( $p$ $>0.05)$.

\section{Persona que cuida a los niños, según comunidad campesina y género}

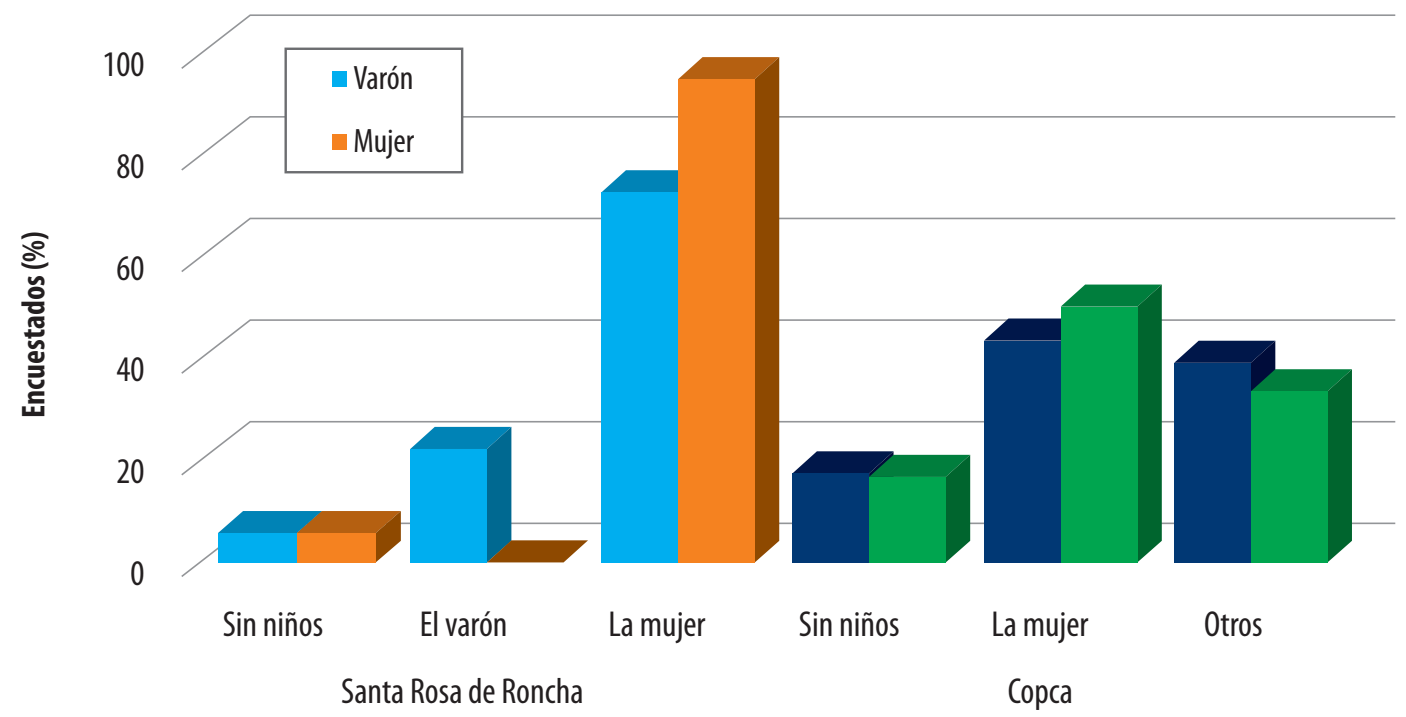


Estos resultados indican que el rol de la mujer y el varón en el cuidado de los niños no es una característica de exclusión social bajo un enfoque de género en las comunidades de Santa
Rosa de Roncha y Copca, con lo cual se rechaza la hipótesis de investigación en lo que se refiere a los roles de la mujer y el varón en el cuidado de grupos vulnerables.

Tabla 10. Persona que cuida los ancianos, según comunidad y campesina y género

\begin{tabular}{clcccr}
\hline \multirow{2}{*}{ Género } & \multirow{2}{*}{ Persona } & \multicolumn{2}{c}{ SR de Roncha } & \multicolumn{2}{c}{ Copca } \\
\cline { 2 - 5 } & Sin ancianos & 6 & 33.3 & 10 & $\%$ \\
\hline \multirow{6}{*}{ Varón } & El varón & 2 & 11.1 & 2 & 43.5 \\
& La mujer & 7 & 38.9 & 5 & 21.7 \\
& Los hijos mayores & 2 & 11.1 & 3 & 13.0 \\
& Otros familiares & 1 & 5.6 & 3 & 13.0 \\
& Total & 18 & 100.0 & 23 & 100.0 \\
\hline \multirow{6}{*}{ Mujer } & Sin ancianos & 7 & 38.9 & 8 & 66.7 \\
& La mujer & 6 & 33.3 & 1 & 8.3 \\
& Los hijos mayores & 2 & 11.1 & 1 & 8.3 \\
& Otros familiares & 3 & 16.7 & 2 & 16.7 \\
& Total & 18 & 100.0 & 12 & 100.0 \\
\hline
\end{tabular}

Roncha: Estadístico chi cuadrado $=0.216$; Valor $p=0.642$

Copca: Estadístico chi cuadrado $=0 ;$ Valor $p=1$

En la tabla 10 y figura 10, se aprecia que en la comunidad de Santa Rosa de Roncha, el 38.9\% de varones y el $33.3 \%$ de mujeres entrevistadas informan que la mujer cuida a los ancianos, el $11,1 \%$ de varones y mujeres manifiestan que lo hacen los hijos mayores.

En la comunidad de Copca, el $21.7 \%$ de varones y el $8.3 \%$ de mujeres manifiestan que la mujer cuida a los ancianos, el $8.7 \%$ de varones dicen que lo hace el varón.

Figura 10.

\section{Persona que cuida a los ancianos, según comunidad campesina y género}

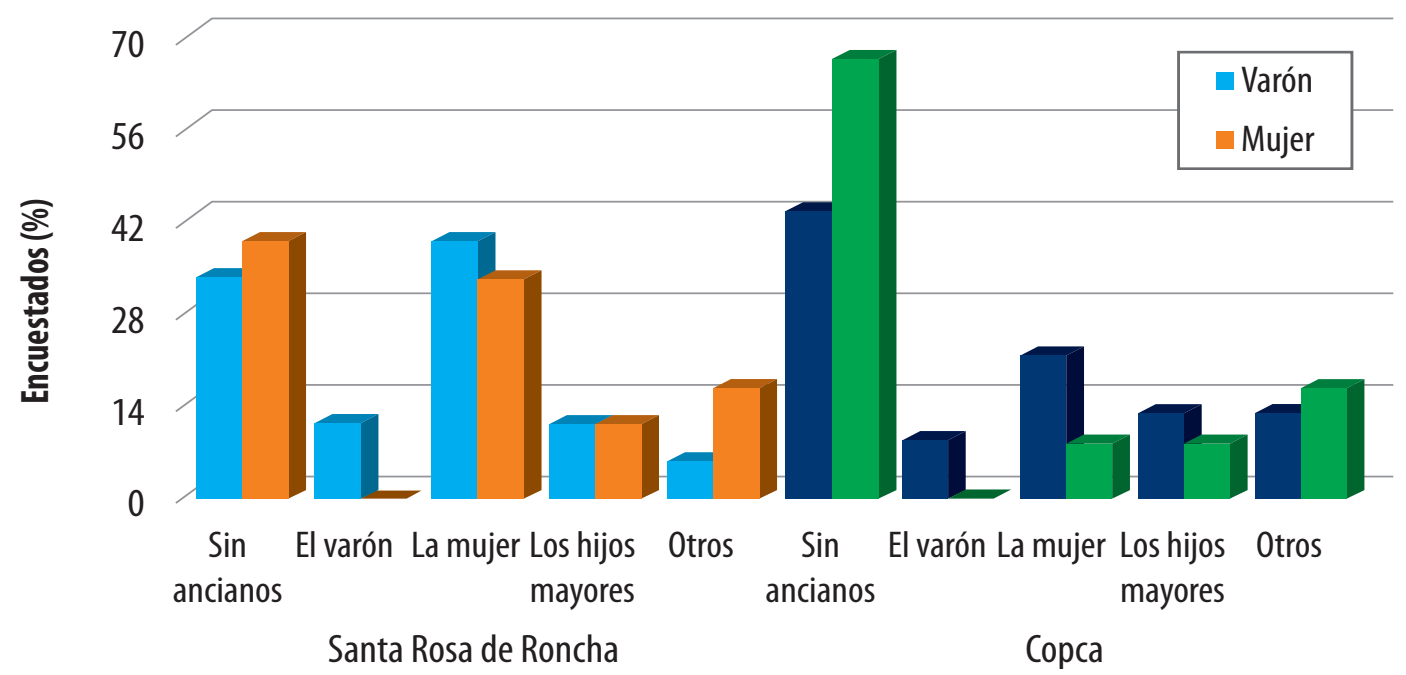


La prueba chi cuadrado para la homogeneidad de muestras revela que en ambas comunidades no existen diferencias significativas en las proporciones de la persona que cuida a los ancianos en los hogares de los entrevistados, de acuerdo con la información de los varones y mujeres $(p>0.05)$.

Estos resultados indican que el rol de la mujer y el varón en el cuidado de ancianos no es una característica de exclusión social bajo un enfoque de género en las comunidades de Santa Rosa de Roncha y Copca, con lo cual se rechaza la hipótesis de investigación en lo que se refiere a los roles de la mujer y el varón en el cuidado de grupos vulnerables.

\section{DISCUSIÓN}

Meneses (2009) señala que la exclusión social se materializa principalmente en el acceso al empleo, remuneraciones, presencia de mujeres en puestos de dirección, entre otros. En la investigación desarrollada se ha encontrado que en las comunidades de Copca y Roncha se concretiza fundamentalmente en el nivel de ingreso.

En efecto, en relación al nivel de ingreso se tiene que éste constituye una primera característica de exclusión, dado que es esta la característica que permite interrelacionar la economía y la satisfacción de las necesidades materiales y espirituales de las personas. En el caso de Roncha el jornal promedio de los varones encuestados es de S/. 43.50 y de las mujeres S/. 29.62 nuevos soles. Mientras que en Copca el jornal promedio de los varones encuestados es de S/. 42.92 y de las mujeres $\mathrm{S} / .35 .17$ nuevos soles.

En torno al empleo precario se tiene que a diferencia del área urbana, ésta no ha alcanzado un comportamiento claramente definido como una característica de exclusión.

Por otra parte, se argumenta que la falta de servicios básicos también constituye otra característica de exclusión social. Al respecto, se ha encontrado que en la comunidad de Roncha, la mayoría de varones $(94 \%)$ y mujeres $(83 \%)$ encuestados responden tener su vivienda construida con material de adobe. De igual modo, en la comunidad de Copca la mayoría de varones (83\%) y mujeres $(67 \%)$ entrevistados dicen tener viviendas construidas de adobe.

Por el que, considerando los resultados de la prueba chi cuadrado para la homogeneidad de muestras se tiene que en ambas comunidades no existen diferencias significativas en las proporciones de viviendas de calidad baja.

Con respecto al índice de hacinamiento de las viviendas en la comunidad de Roncha, se tiene que éste es de 1 a 2 personas por habitación. Mientras que en la comunidad de Copca, el índice de hacinamiento es de 2 a más personas por habitación.

Luego de aplicado la prueba paramétrica de la F de Fisher para la homogeneidad de varianza se encuentra que en ambas comunidades no hay diferencias entre las varianzas del índice de hacinamiento en las viviendas de los varones y mujeres. Por el que, el índice de hacinamiento en la vivienda no es una característica de exclusión social bajo el enfoque de género en las comunidades de Roncha y Copca.

En relación a que las mujeres continúan asumiendo principalmente la mayor parte del trabajo doméstico y el cuidado de la familia (Meneses, 2009), en el estudio se corrobora que en efecto, tanto en Copca como en Roncha las mujeres son las que asumen la tarea del cuidado del hogar ( $83 \%$ y $82 \%)$, los niños $(89 \%$ y $78 \%$ ) y adultos mayores ( $94 \%$ y $50 \%$ ).

Con respecto a la toma de decisiones y la participación política, Meneses (2009) señala que en cuanto a la participación de la mujer aún se tiene una brecha muy importante. En la investigación desarrollada, se ha encontrado que en cuanto al desempeño de cargos comunales o públicos en Copca y Cochangará estadísticamente no existe mayor diferencia entre hombres y mujeres. Al respecto, se ha encontrado que en la comunidad de Roncha el $22 \%$ de varones y el $11 \%$ de mujeres entrevistados respondieron haber desempeñado algún cargo en la comunidad. Mientras que en la comunidad de Copca, el $4 \%$ de varones y ninguna mujer dicen haber desempeñado algún cargo en la comunidad. Aplicado la prueba chi cuadrado para 
la homogeneidad de muestras se tiene que en ambas comunidades no existen diferencias significativas en las proporciones de desempeño de algún cargo en la comunidad.

\section{CONCLUSIONES}

1. En las comunidades campesinas de Santa Rosa de Roncha y de Copca, el nivel de ingreso constituye una primera característica de exclusión social bajo un enfoque de género. En efecto, considerando la prueba paramétrica $U$ de Mann Whitney para la homogeneidad de muestras se tiene que en ambas comunidades existen diferencias significativas en las medias del jornal diario de los varones y mujeres encuestados como peón agrícola favorable a los varones.

2. Con respecto al empleo precario, se ha encontrado que en Copca representa otra característica de exclusión social bajo el enfoque de género; más no así, en Santa Rosa de Roncha. En efecto, considerando la prueba chi cuadrado para la homogeneidad de muestras se tiene que en la comunidad de Roncha, no existen diferencias en las proporciones del empleo precario de los encuestados. En cambio, en la comunidad de Copca, si presenta diferencias en las proporciones del empleo precario de los encuestados varones y mujeres.

3. En relación a los servicios básicos como característica de exclusión social, se ha encontrado que en la comunidad de Roncha, la mayoría de varones (94\%) y mujeres (83\%) respondieron tener su vivienda construida con material rústico (adobe).

Ahora bien, considerando los resultados de la prueba chi cuadrado para la homogeneidad de muestras se encuentra que en ambas comunidades no existen diferencias significativas en las proporciones de viviendas de baja calidad. Por el que se puede concluir que la calidad baja (materiales de construcción) de las viviendas no constituye una característica de exclusión bajo el enfoque de género en las comunidades en referencia.

4. El desempeño de cargos comunales al interior de la comunidad, también es considerado una característica de exclusión so- cial. Sobre el particular se ha encontrado en la comunidad de Roncha que el $22 \%$ de varones y el $11 \%$ de mujeres entrevistadas respondieron haber desempeñado algún cargo en la comunidad. Mientras que en la comunidad de Copca el $4 \%$ de varones y ninguna mujer refieren haber desempeñado cargo alguno en la comunidad.

Aplicado la prueba chi cuadrado para la homogeneidad de muestras se tiene que en ambas comunidades no existen diferencias en las proporciones de desempeño de algún cargo en la comunidad. Por tanto, se concluye que en cuanto al rol de la mujer y del varón en las comunidades rurales en estudio no es una característica de exclusión social bajo el enfoque de género.

5. Se ha encontrado en las comunidades de Roncha y Copca que la dependencia económica de los niños no es una característica de exclusión social bajo un enfoque de género de acuerdo a los resultados obtenidos mediante la prueba chi cuadrado que indica que no existe diferencias en las proporciones de las personas que cuidan del mantenimiento de los niños.

6. Igualmente, en relación al cuidado de los adultos mayores se ha encontrado que de acuerdo a la prueba chi cuadrado para la homogeneidad de muestras no existen diferencias en las proporciones de las personas que cuidan a los adultos mayores en ambas comunidades.

\section{RECOMENDACIONES}

1. Desarrollar investigaciones sobre el tema de la exclusión social bajo un enfoque de género a nivel explicativo y de carácter interdisciplinario, cuyos resultados y conclusiones permitan proponer políticas inclusivas en el área rural.

2. Replicar nuevas investigaciones en el área rural a fin de confirmar la diferencia de las características de la exclusión social bajo un enfoque de género en el área rural con respecto al área urbano.

3. En el diseño metodológico, mejorar la calidad del instrumento de recopilación de la data (estructura de la encuesta). 


\section{REFERENCIAS BIBLIOGRÁFICAS}

1. Bel Adell, Carmen (2003). Exclusión social: orígenes y características. Universidad de Murcia, España.

2. Brumel, J. (2012). Recuperado de http: //www. Fao. org/docrep/004/x29195/ x2919s04.htm

3. Cabrera H., Luis (2005). Pobreza y exclusión social. España, Fundación Luis Espinal.

4. Cacifúa, E. y Davis, S.H. (2000). Introducción: Pobreza y exclusión social en América Latina y el Caribe, en E. Cacifúa, C. Sojo y S.H. Davis (edit.).

5. Comisión de las Comunidades Europeas (1993). Informe secundario: 6.-6.- Exclusión social, pobreza y otros problemas sociales en la Comunidad 7.- Europea, ISE$C / B / 11 / 93$, Luxemburgo: Oficina para las publicaciones oficiales de las comunidades europeas.

6. García Ełal, Mauricio (2005). El campo y la ciudad. Sociedad rural y 9.-cambio social, Madrid.

7. Kichert N., Adrían (1997). Las condiciones de vida de los hogares pobres. Pobre- za y género, Caritas Española, Fundación FOESSA.

8. Martinez R., Ma Asunción (2001). Género, pobreza y exclusión: diferentes conceptualizaciones y políticas públicas, en Tostosa, José María (coord..), Pobreza y perspectivas de género. Icaria, Barcelona.

9. Meneses F., Carmen (2009). Género, desigualdad e inclusión. Madrid.

10. Pérez S., J.P. y Minor Mora S. (2006). De la pobreza a la exclusión social. La persistencia de la miseria en Centroamérica. Pdf. FLACSO-Costa Rica, San José

11. Exclusión Social (1994). Hacia una Europa de las solidaridades intensificando la lucha contra la exclusión social y promoviendo la integración.

12. PNUD (2004). Como elaborar una estrategia de género para una oficina de país. El Salvador.

13. Ulrich Beck (2002). Pobreza y exclusión. Consejo Superior de Investigación Científica. Instituto de Estudios Sociales, Madrid.

14. Valdivia, Nestor (2011). Antecedentes exclusión social por enfoque de género. 